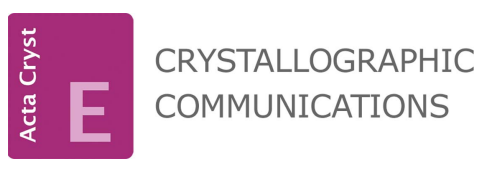

ISSN 2056-9890
Received 18 May 2020

Accepted 5 June 2020

Edited by M. Weil, Vienna University of Technology, Austria

Keywords: phenol; hydrate; neutron powder diffraction; thermal expansion; DFT; Hirshfeld surface analysis.

CCDC reference: 2008230

Supporting information: this article has supporting information at journals.iucr.org/e

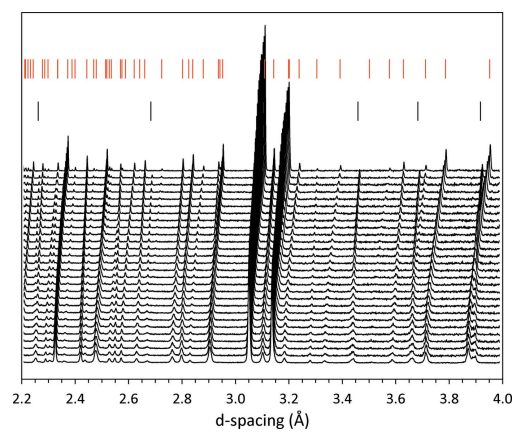

\section{Phenol hemihydrate: redetermination of the crystal structure by neutron powder diffraction, Hirshfeld surface analysis and characterization of the thermal expansion}

\author{
A. Dominic Fortes*
}

ISIS Neutron \& Muon Spallation Facility, Rutherford Appleton Laboratory, Harwell Science and Innovation Campus, Chilton, Oxfordshire, OX11 0QX, England. *Correspondence e-mail: dominic.fortes@stfc.ac.uk

Phenol hemihydrate, $\mathrm{C}_{5} \mathrm{H}_{5} \mathrm{OH} \cdot 0.5 \mathrm{H}_{2} \mathrm{O}$, crystallizes in the space group $P b c n, Z=$ 8. The previously published crystal structure [CSD refcode PHOLHH; Meuthen \& von Stackelberg (1960). Z. Elektrochem. 64, 387-390] is shown to be incorrect. Pairs of phenol molecules, related by an inversion centre, are bridged by one water molecule via $\mathrm{O}-\mathrm{H} \cdots \mathrm{O}$ hydrogen bonds; an extended $R_{4}^{4}(8)$ hydrogenbonded motif links these inversion dimers into chains parallel to the $c$ axis. Packing of the chains is achieved by weaker T-shaped $\mathrm{C}-\mathrm{H} \cdots \pi$ interactions between nearest neighbour phenol molecules in the $b c$ plane. Analysis of the thermal expansion and parameterization with a Debye model in terms of the linear elastic moduli shows that the $c$ axis is $\sim 3$ times stiffer than the two orthogonal directions.

\section{Chemical context}

Phenol is the simplest aromatic alcohol; as such, it is one of the most straightforward systems in which to study the competition between medium-strength $\mathrm{O}-\mathrm{H} \cdots \mathrm{O}$ hydrogen bonds, $\pi \cdots \pi / \mathrm{C}-\mathrm{H} \cdots \pi$ interactions and steric effects on packing in the solid state (Zavodnik et al., 1988: Allan et al., 2002). However, there are sparse structural data on compounds formed solely between phenol and simple polar molecules such as water or ammonia. The binary phenol-water system is characterized by a wide region of liquid immiscibility (Smith, 1932); liquids on the phenol-rich side exhibit a propensity to supercool and crystallize solid phenol rather than an intermediate hydrated compound. Consequently, early reports of a hemihydrate by Calvert (1865) proved difficult to reproduce (Alexeev, 1883: Paternò \& Ampola, 1897). Rapid freezing of stoichiometric liquids with dry ice or liquid air was found to reproducibly form seed crystals of the hemihydrate for structural characterization (Smits \& Maarse, 1911: Rhodes \& Markley, 1921). Although the hydrate melts quite close to room temperature $\left(T_{\mathrm{m}}=289 \mathrm{~K}\right)$, the non-H-atom crystal structure of phenol hemihydrate was not reported for several more decades (Meuthen \& von Stackelberg, 1960; CSD refcode PHOLHH).

As part of a wider study into the structures of complexes formed between simple alcohols and water or ammonia (Fortes, 2019), neutron powder-diffraction data were collected from a perdeuterated analogue of phenol hemihydrate with the simple objective of determining the hydrogen-atom positions. However, the intensities of the observed Bragg peaks differed so greatly from the calculated intensities as to be 


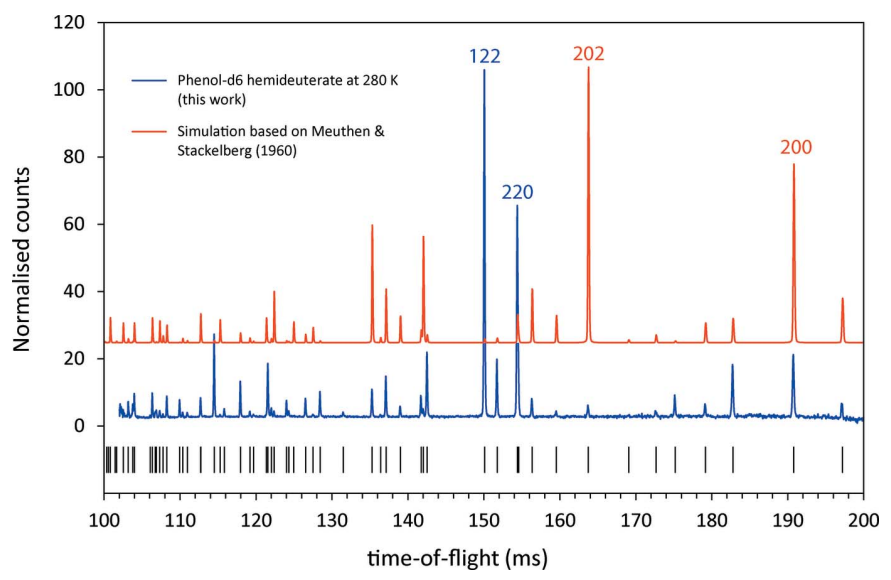

Figure 1

Time-of-flight (TOF) data collected in HRPD's backscattering detectors over the range $100-200 \mathrm{~ms}$ at $280 \mathrm{~K}$ (blue) compared with a simulated diffraction pattern (red) based on the structure model of Meuthen \& von Stackelberg (1960). The latter structure was 'completed' with geometrically positioned deuterons $1.080 \AA$ from, and co-planar with, the phenyl carbons, a deuteron placed $0.990 \AA$ from the hydroxyl oxygen along the $\mathrm{O}-\mathrm{O}$ vector that gave the smallest $\mathrm{C}-\mathrm{C}-\mathrm{O}-\mathrm{D}$ torsion angle; the symmetry-unique water deuteron was placed $0.990 \AA$ from the water oxygen along the remaining $\mathrm{O}-\mathrm{O}$ vector. In the experimentally observed diffraction pattern, the strongest Bragg peaks in this TOF range are 122 and 220. In contrast, the intensity of 122 from the simulated pattern is extremely weak and 202 has the greatest intensity instead.

irreconcilable with a structure that was generally correct but merely incomplete (Fig. 1). In conclusion, Meuthen \& von Stackelberg's structure model is incorrect; the neutron powder data were used to determine the correct structure, as reported below.

\section{Structural commentary}

Phenol hemihydrate, $\left(\mathrm{C}_{5} \mathrm{H}_{5} \mathrm{OH}\right) \cdot 0.5 \mathrm{H}_{2} \mathrm{O}$, forms an inversion dimer, such that there is only one symmetry-independent phenol molecule per formula unit with atoms on general positions $8 d$; the bridging water molecule occupies the higher symmetry $4 c$ site associated with a twofold rotation axis (Fig. 2). The phenyl rings are flat, atoms C1-C6 lying no more than $0.005 \AA$ from a least-squares plane (LSP) fitted through the carbon atoms; hydrogen atoms D1-D5 and the hydroxyl oxygen, O1, lie within $0.041 \AA$ of the same LSP. The hydroxyl

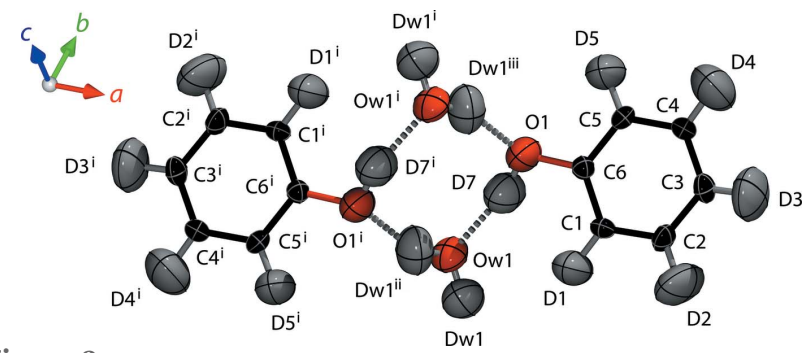

Figure 2

Phenol hemihydrate's asymmetric unit (right) and the inversion-related atoms required to form the dimer (left); the inversion centre is located in the middle of the ring. Displacement ellipsoids are drawn at the $50 \%$ probability level. [Symmetry codes: (i) $-x, 1-y, 1-z$; (ii) $-x, y, \frac{1}{2}-z$; (iii) $\left.x, 1-y, \frac{1}{2}+z\right]$ (a)

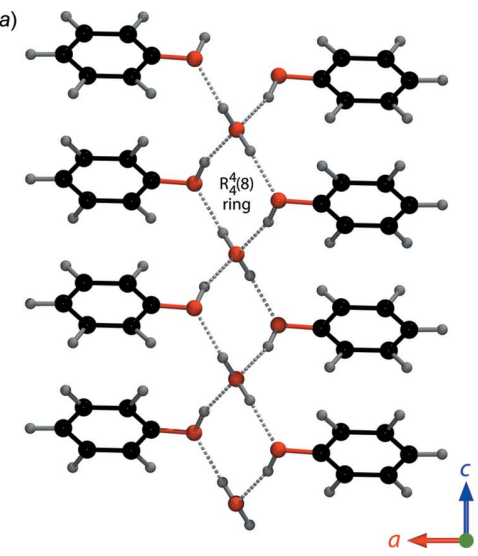

(b)

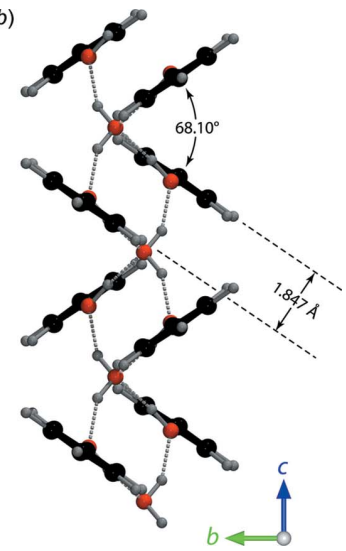

Figure 3

(a) The dimer shown in Fig. 2 extends as a chain parallel to the $c$ axis. (b) The chain viewed parallel to the $c$ axis reveals the offset of the phenyl rings in each dimer and the hinge angle between successive dimers along the chain.

deuteron, D7, lies $0.192 \AA$ out of the LSP, leading to a dihedral angle $\mathrm{C} 1-\mathrm{C} 6-\mathrm{O} 1-\mathrm{D} 7$ of $17.1(6)^{\circ}$. The hydroxyl moiety is in a trigonal coordination, both donating and accepting one hydrogen bond from neighbouring water molecules. These hydrogen bonds generate a rhombic motif involving two water molecules and two hydroxyl moieties (Fig. 2), which may be described by the graph-set notation $R_{4}^{4}(8)$. The water molecules are in a tetrahedral coordination, connecting the $R_{4}^{4}(8)$ rings to form an infinite chain of inversion dimers extending parallel to the $c$ axis (Fig. 3a). Inversion-related phenol pairs sit on planes with a vertical separation of $1.847 \AA$ and these are in turn canted alternately along the $c$ axis so as to form sheets that are co-planar with (011) and (011) (interplanar angle $=71.92^{\circ}$ ). Individual phenyl rings are tilted slightly with respect to these sheets such that the hinge angle between directly adjacent pairs of phenol LSPs is reduced to $68.10^{\circ}$ (Fig. 3b).

More importantly, the $\mathrm{C}-\mathrm{O}$ bonds of the two symmetryrelated phenol molecules involved in the dimer are approximately co-aligned with the $a$ axis of the crystal, and this marks the principal point of difference with the structure model reported by Meuthen \& von Stackelberg (1960). They correctly inferred the coordination environment of the $\mathrm{O}$ atoms and used trial-and-error methods [complemented by a Fourier map projected on (001)] to determine the arrangement of the phenyl rings. Hence, their model contains the same chains of corner-linked four-sided rings extending parallel to the $c$ axis, but it differs from the correct structure by having the inversion dimers aligned approximately along the body diagonals of the unit cell. This leads to a significantly different packing of the phenol molecules, as outlined below.

\section{Supramolecular features}

Intermolecular hydrogen-bond lengths and angles are reported in Table 1. Fig. 4 compares the unit-cell contents, viewed along each crystallographic axis, between the structure published by Meuthen \& von Stackelberg (1960) and this 
Table 1

Hydrogen-bond geometry $\left(\AA,^{\circ}\right)$.

$C g$ is the centroid of the $\mathrm{C} 1-\mathrm{C} 6$ ring located at $(0.310,0.634,0.451)$.

\begin{tabular}{lllll}
\hline$D-\mathrm{H} \cdots A$ & $D-\mathrm{H}$ & $\mathrm{H} \cdots A$ & $D \cdots A$ & $D-\mathrm{H} \cdots A$ \\
\hline O1-D7 $\cdots$ Ow & $0.97(1)$ & $1.85(1)$ & $2.793(7)$ & $165(1)$ \\
Ow-Dw $\cdots$ O $1^{\text {ii }}$ & $0.98(1)$ & $1.83(1)$ & $2.746(5)$ & $154(1)$ \\
C1-D1 $\cdots C g^{\text {iii }}$ & $1.08(1)$ & 3.15 & 3.844 & 123 \\
C2-D2 $\cdots C g^{\text {iii }}$ & $1.06(1)$ & 3.29 & 3.897 & 118 \\
C5-D5 $\cdots C g^{\text {iv }}$ & $1.06(1)$ & 2.89 & 3.719 & 136 \\
\hline
\end{tabular}

Symmetry codes: (i) $-x, y,-z+\frac{1}{2}$; (ii) $-x,-y+1,-z+1$; (iii) $x,-y+1, z-\frac{1}{2}$; (iv) $-x+\frac{1}{2},-y+\frac{3}{2}, z+\frac{1}{2}$.

work. As noted above, Meuthen \& von Stackelberg constructed a Fourier map projected on (001) and, clearly, the molecular structure viewed along $c$ is quite similar to that obtained here. However, the orientation of the phenol molecules out of the (001) plane differs such that the (100) and (010) projections are completely different.

Phenol molecules from one of the chains shown in Fig. $3 a$ interlock with those of an adjacent chain. The resultant slab of nearest-neighbour phenol molecules lies in the $b c$ plane, a cross-section of which is represented by the grey rectangle in Fig. 4a. An isolated view of the slab along the $a$ axis (Fig. 5a) reveals a rhombic array of $\mathrm{T}$-shaped $\mathrm{C}-\mathrm{H} \cdots \pi$ interactions with a mean separation of $4.93 \AA$ between molecular centres. The equivalent slab of nearest-neighbour phenol molecules in Meuthen \& von Stackelberg's structure model lies in the $a c$ plane (grey rectangle in Fig. $4 b$ ); when viewed along the $b$ axis (Fig. 5b), a skewed hexagonal array of molecular centres is found in which the interactions involve both T-shaped $\mathrm{C}-$ $\mathrm{H} \cdots \pi$ contacts and offset $\pi-\pi$ stacking. The mean distance between molecular centres is $4.69 \AA$.

\section{Hirshfeld surface analysis}

A useful method of analysing and comparing inter- and intramolecular interactions is by calculation of a Hirshfeld surface (Spackman \& Jayatilaka, 2009) and derivation of two- (a)

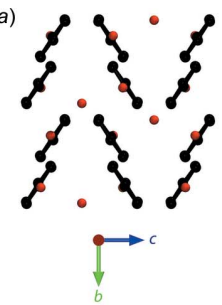

(b)

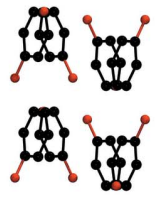

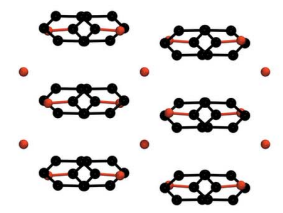
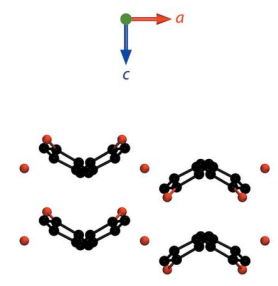
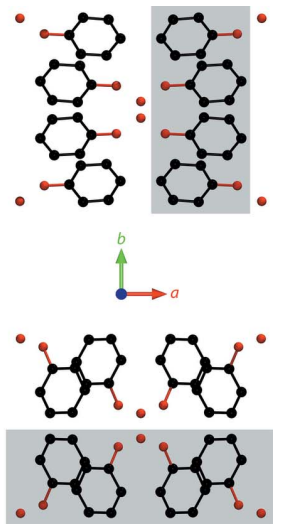

Figure 4

Molecular packing in phenol hemihydrate $(a)$ from this work and $(b)$ from Meuthen \& von Stackelberg (1960). Hydrogen atoms are omitted for clarity. Shaded rectangles indicate sections through slabs of closestpacked phenol molecules, which are drawn explicitly in Fig. 5. dimensional fingerprint plots (McKinnon et al., 2007). These have been computed for the structure obtained in this work, for Meuthen \& von Stackelberg's structure model (PHOLHH) and for solid phenol (PHENOL03; Zavodnik et al., 1988) using CrystalExplorer 17.5 (Turner et al., 2017). The $d_{\text {norm }}$ plot for the correct structure of phenol hemihydrate (Fig. $6 a$ ) was found via calculation of the external $\left(d_{\mathrm{e}}\right)$ and internal $\left(d_{\mathrm{i}}\right)$ distances between pairs of nuclei with a scaled colour of -0.6026 a.u. (red) to 1.1002 a.u. (blue). A corresponding plot of the shape-index was generated in the range of -1.0 to 1.0 a.u. (Fig. $6 b$ ).

Red areas on the Hirshfeld surface indicate contacts that are shorter than the sum of the van der Waals radii and blue areas show where the contacts are longer than the vdW sum. Clearly, there are red patches on the Hirshfeld surface that correspond only with the $\mathrm{O}-\mathrm{H} \cdots \mathrm{O}$ hydrogen bonds. For Meuthen \& von Stackelberg's structure model (see Fig. S1 in the supporting information) there are additional red patches in proximity to some of the ring hydrogen atoms, indicating some very short $\mathrm{C}-\mathrm{H} \cdots \mathrm{O}$, and even $\mathrm{H} \cdots \mathrm{H}$, interactions. Weaker $\mathrm{C}-\mathrm{H} \cdots \pi$ interactions are more clearly elucidated from the shape-index plot (Fig. 6b), where the strongly negative (red) regions delineate specific labelled contacts.

(a)

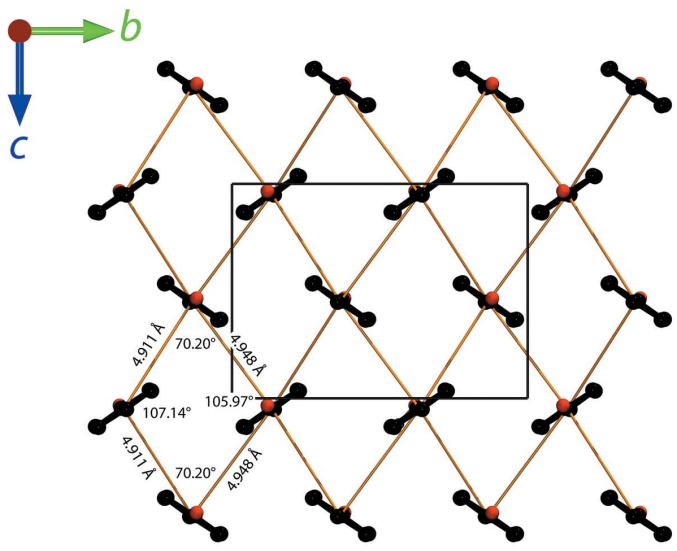

(b)

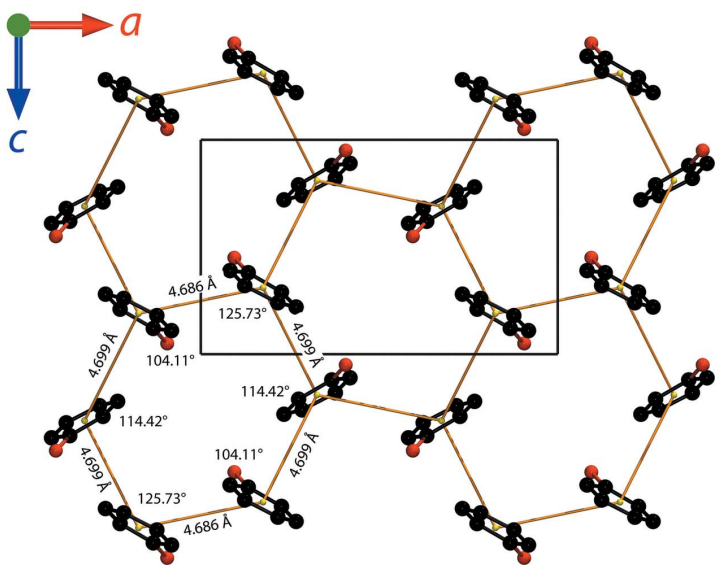

Figure 5

Illustration of the nearest-neighbour interactions in the slabs of interlocking phenol molecules $(a)$ from this work and $(b)$ from Meuthen \& von Stackelberg (1960). Hydrogen atoms are omitted for clarity and the network of molecular centres is indicated by solid yellow rods. 

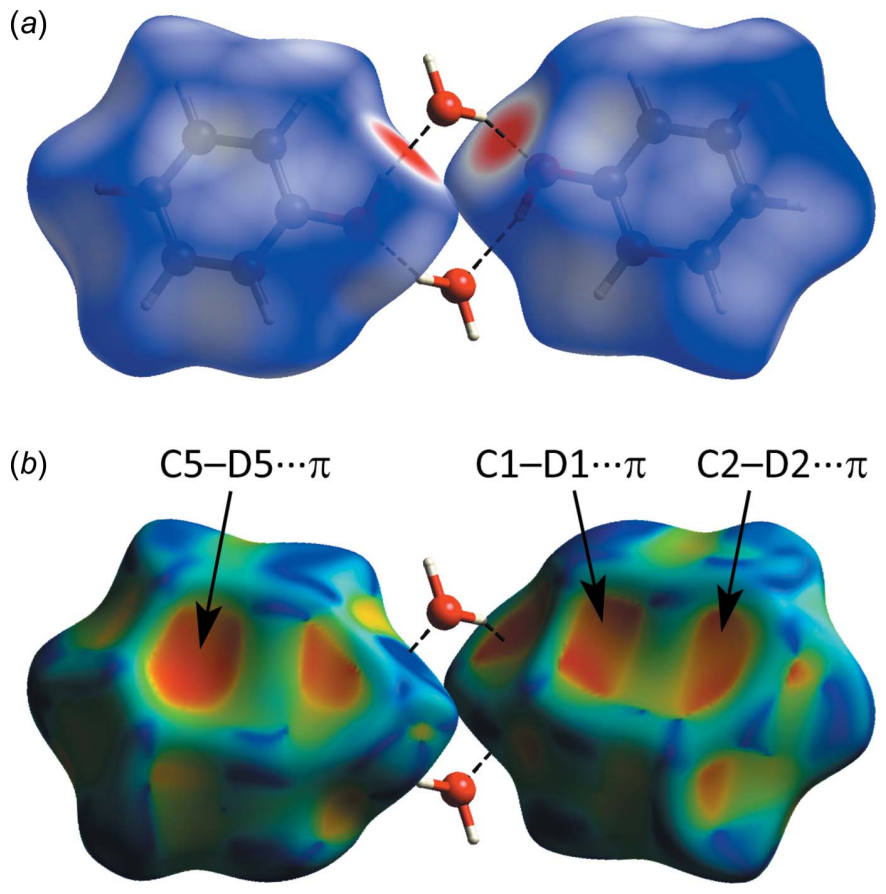

Figure 6

Hirshfeld surfaces of phenol hemihydrates, superimposed on the inversion dimer shown in Fig. 2. Surfaces are shaded by $(a) d_{n o r m}$ value and $(b)$ the shape-index. Regions of important intermolecular contacts referred to in the text are labelled (see also Table 1).

Two-dimensional fingerprint plots are shown in Fig. 7. The $\mathrm{O} \cdots \mathrm{H} / \mathrm{H} \cdots \mathrm{O}$ hydrogen-bonded contacts appear as the two sharply pointed regions spreading to the top right from $d_{\mathrm{i}}+d_{\mathrm{e}}$ $\simeq 1.82 \AA$; these represent $15.2 \%$ of the Hirshfeld surface
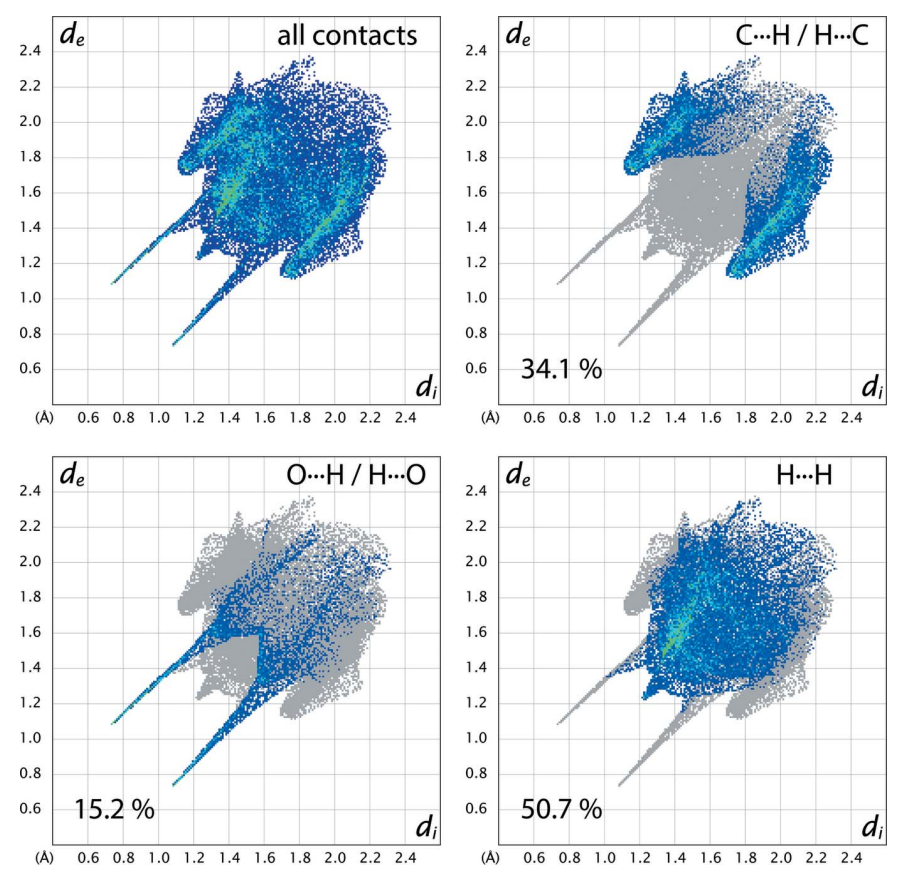

Figure 7

Two-dimensional fingerprint plots showing the distribution of interatomic contacts on the Hirshfeld surface of the phenol molecule in phenol hemihydrate. around the phenol molecule. The next largest contribution to the surface area $(34.1 \%)$ comes from $\mathrm{C} \cdots \mathrm{H} / \mathrm{H} \cdots \mathrm{C}$ interactions, which appear on the fingerprint plots as two approximately symmetrical round-tipped 'wings' spreading to the top right from $d_{\mathrm{i}}+d_{\mathrm{e}} \simeq 2.82 \AA$, and these represent the T-shaped $\mathrm{C}-\mathrm{H} \cdots \pi$ contacts. The balance of the Hirshfeld surface area $(50.7 \%)$ comes exclusively from $\mathrm{H} \cdots \mathrm{H}$ contacts. There are no contributions from $\mathrm{C} \cdots \mathrm{C}, \mathrm{C} \cdots \mathrm{O}$ or $\mathrm{O} \cdots \mathrm{O}$ contacts.

The fingerprint plots for solid phenol (supplementary Fig. S2) are strikingly similar to those for phenol hemihydrate. There are three symmetry-inequivalent phenol molecules in the asymmetric unit of phenol, and the average surface area contributions for the various contacts are: $\mathrm{O} \cdots \mathrm{H} / \mathrm{H} \cdots \mathrm{O}=$ $15.9 \% ; \mathrm{C} \cdots \mathrm{H} / \mathrm{H} \cdots \mathrm{C}=33.1 \% ; \mathrm{H} \cdots \mathrm{H}=49.9 \% ; \mathrm{C} \cdot \mathrm{C}=0.5 \%$; $\mathrm{C} \cdots \mathrm{O} / \mathrm{O} \cdots \mathrm{C}=0.6 \%$. Despite the substantial influence of $\mathrm{O}-$ $\mathrm{H}$... O hydrogen bonds on the packing, the principal interaction between phenyl rings in solid phenol is still via T-shaped $\mathrm{C}-\mathrm{H} \cdots \pi$ contacts.

By contrast, the fingerprint plots for Meuthen and von Stackelberg's phenol hemihydrate structure model (supplementary Fig. S3) display strong indicators that their solution is not correct. In particular, there are some very short $\mathrm{H} \cdots \mathrm{H}$ contacts, with $d_{\mathrm{i}}+d_{\mathrm{e}} \simeq 1.39 \AA$, and these comprise $55.3 \%$ of the surface area. As it is a matter of trivial geometry to calculate on paper the positions of the ring hydrogens and then to compute interatomic distances, it should have been obvious from the outset that their structure was incorrect. The remainder of the contributions to the Hirshfeld surface area are as follows: $\mathrm{O} \cdots \mathrm{H} / \mathrm{H} \cdots \mathrm{O}=14.3 \% ; \mathrm{C} \cdot \mathrm{H} / \mathrm{H} \cdots \mathrm{C}=26.6 \%$; $\mathrm{C} \cdots \mathrm{C}=3.3 \% ; \mathrm{C} \cdots \mathrm{O} / \mathrm{O} \cdots \mathrm{C}=0.5 \%$. Note that the significant C. . C contribution is due to the offset $\pi-\pi$ stacking that is apparent from Fig. $5 a$.

\section{Thermal expansion}

Lattice parameters of phenol hemihydrate determined between 10 and $280 \mathrm{~K}$ are reported in supplementary Table S1 and plotted in supplementary Fig. S4. Precision at low temperatures is significantly poorer due to substantial strain broadening of the Bragg peaks. These data have been fitted with a second-order Grüneisen approximation to the zeropressure equation of state [Equation (1)]. In this approximation, the thermal expansion is considered equivalent to elastic strain such that,

$$
V(T)=V_{0}[1+E(T) / Q-b E(T)]
$$

where $V_{0}$ is the unit-cell volume at zero pressure, $b=$ $1 / 2\left(K_{0}^{\prime}-1\right)$ and $Q=\left(V_{0} K_{0} / \gamma\right) ; K_{0}$ is the zero pressure isothermal bulk modulus, $K_{0}^{\prime}$ is its first derivative with respect to pressure, and $\gamma$ is the thermal Grüneisen parameter. The internal energy due to lattice vibrations, $E(T)$, is then determined via a Debye model:

$$
E(T)=\left(9 n k_{\mathrm{B}} T\right) /\left(\theta_{D} / T\right)^{3} \int_{0}^{\left(\theta_{D} / T\right)} x^{3} /\left(e^{x}-1\right) d x
$$


where $\theta_{\mathrm{D}}$ is the Debye temperature, $n$ is the number of atoms per formula unit, and $k_{\mathrm{B}}$ is the Boltzmann constant; the integral term is evaluated numerically. In order to be dimensionally correct, the individual lattice parameters were fitted as $a^{3}, b^{3}$ and $c^{3}$; the fit parameters (along with a fit to the unitcell volume) are given in supplementary Table S2. The values of $K_{0} / \gamma$ reported for each axis therefore correspond with, e.g., $K_{a} / \gamma=-a^{3}\left(\mathrm{~d} P / \mathrm{d} a^{3}\right)$.

Supplementary Fig. S5 shows the linear and volume thermal expansion coefficients as a function of temperature. The thermal expansivity of the $a$ axis $\left(\alpha_{1}\right)$ differs substantially from that of $b$ or $c\left(\alpha_{2}\right.$ and $\alpha_{3}$, respectively), reflected in their very different Debye temperatures and their derived elastic moduli. The linear incompressibility of the $c$ axis (assuming no anisotropy of $\gamma$ ) is almost three times larger than the two orthogonal directions, although this remains to be confirmed by any high-pressure studies. The observed behaviour along $a$ and $b$ is due to weaker dispersion interactions between and within the nearest-neighbour slabs (Fig. 5a) whereas the behaviour along $c$ is governed by $\mathrm{O}-\mathrm{H} \cdots \mathrm{O}$ hydrogen bonds in the chains of corner-linked $R_{4}^{4}(8)$ rings.

\section{Database survey}

Searches of the Cambridge Structural Database (CSD Version 5.41, March 2020 update; Groom et al., 2016) were carried out to identify structures with geometrically similar $\mathrm{O} \cdots \mathrm{O}$ hydrogen-bonding motifs and similar distorted T-shaped $\mathrm{C}-$ $\mathrm{H} \cdots \pi$ motifs.

Square rings comprised of two alcohol $\mathrm{O}-\mathrm{H}$ groups and two water molecules are comparatively uncommon in organic crystals; examples include CSD refcodes KONTIQ (Demirtaş et al., 2011), AYOPIO (Chantrapromma et al., 2011), CERYIK (Zhang et al., 2018) and VABKOA (Li et al., 2010). However, the compound most closely related structurally to phenol hemihydrate that contains this motif is TMBUOL $(2,3,3-$ trimethyl butan-2-ol hemihydrate; Pachler \& von Stackelberg, 1963).

The rhombic motif of $\mathrm{C}-\mathrm{H} \cdots \pi$ interactions generated over 2800 hits in the CSD search, of which the most interesting are the closely related mono-substituted benzenes: chlorobenzene (MCBENZ; Biswas, 1958; André et al., 1971; Nath \& Naumov, 2015), bromobenzene (ZZZSPA; Biswas, 1958) and iodobenzene (REKYAI; Merz, 2006). Each of these crystallizes in space-group type Pbcn and adopts a near identical molecular packing of the phenyl rings to that observed in phenol hemihydrate. The lattice parameters of $\mathrm{C}_{6} \mathrm{H}_{6} \mathrm{Cl}$ are very similar to $\left(\mathrm{C}_{6} \mathrm{H}_{5} \mathrm{OH}\right) \cdot 0.5 \mathrm{H}_{2} \mathrm{O}$ and the principal difference on substitution of $\mathrm{Br}$ and $\mathrm{I}$ is an increase in the length of the $a$ axis as the length of the carbon-halogen bond increases, these being roughly co-aligned with $a$ in the same fashion as the $\mathrm{C}-\mathrm{O}$ bond in phenol hemihydrate. Similarly, both thiophenol (JUJPEL; Thomas et al., 2015) and selenophenol (JUJPAH; Thomas et al., 2015) adopt the same packing as the phenol molecule in phenol hemihydrate. These are reported in space group Pnab rather than the conventional setting of Pbcn, otherwise the only meaningful difference is the presence of
$\mathrm{S}-\mathrm{H} \cdots \mathrm{S}$ or $\mathrm{Se}-\mathrm{H} \cdots \mathrm{Se}$ chains long the crystal's $a$ axis instead of rings of $\mathrm{O}-\mathrm{H} \cdots \mathrm{O}$ hydrogen bonds via $\mathrm{H}_{2} \mathrm{O}$.

It is worth adding that no matches to the phenol packing motif in Meuthen \& von Stackelberg's (1960) structure were found in the CSD.

\section{DFT geometry relaxations}

Zero-pressure athermal geometry optimizations of the phenol hemihydrate structure were performed using Density Functional Theory, DFT, and the plane-wave pseudopotential method (Hohenberg \& Kohn, 1964: Kohn \& Sham, 1965). The calculations were implemented in CASTEP v 17.2 (Payne et al., 1992: Segall et al., 2002: Clark et al., 2005) in conjunction with the analysis tools in the Materials Studio software package. Ultrasoft pseudopotentials with a basis-set cut-off of $1200 \mathrm{eV}$ and a $2 \times 2 \times 3 \vec{k}$-point grid $\left(\sim 0.04 \AA^{-1}\right.$ reciprocal lattice spacing) were required to achieve convergence of better than $1 \times 10^{-2} \mathrm{GPa}$ in the stress and better than $1 \times 10^{-3} \mathrm{eV}$ per atom in total energy. The 'PBE' gradientcorrected functional (Perdew et al., 1996, 1997) was used in conjunction with both the Grimme (G06) dispersion correction (Grimme, 2006), the Tkatchenko \& Scheffler (TS) dispersion correction (Tkatchenko \& Scheffler, 2009) and the Many-Body Dispersion (MBD) correction (Tkatchenko et al., 2012).

Structural relaxations were begun from the experimentally determined crystal structure using the BFGS method (Pfrommer et al., 1997). These were considered to have converged when the forces on each atom were less than $5 \times 10^{-3} \mathrm{eV} \AA^{-1}$ and each component of the stress tensor was smaller than $0.005 \mathrm{GPa}$.

Supplementary Tables S4 and S5 report both the inter- and intramolecular distances and angles found in the PBE + MBD, $\mathrm{PBE}+\mathrm{TS}$ and $\mathrm{PBE}+\mathrm{G} 06$ simulations. In each case, the internal geometry of the phenol and the water molecules are nearly identical. However, the intermolecular contacts differ substantially between the G06 and TS-based (TS \& MBD) dispersion corrections, leading to large deviations in the calculated athermal lattice parameters from the observed $10 \mathrm{~K}$ unit-cell dimensions. PBE + TS agrees with the experimental values much more closely than PBE + G06, as expected on the basis of a recent computational survey (Binns et al., 2014). Whilst PBE + MBD gives marginally more accurate intermolecular distances and matches the $10 \mathrm{~K}$ unit-cell volume extremely well, the axial ratios are less accurate than PBE + TS. The structures obtained from the three zero-pressure geometry optimizations are provided in the electronic supplementary information as a CIF.

\section{Measurement, structure solution and refinement}

Crystal data, data collection and structure refinement details are summarized in Table 2. Neutron powder diffraction data were collected from the sample, mounted in a Closed-Cycle Refrigerator (CCR) on the High Resolution Powder Diffractometer (HRPD) at the ISIS spallation neutron source 
(Ibberson, 2009). Initial examination of the specimen at $250 \mathrm{~K}$ revealed the presence of $\sim 3$ wt. $\% \mathrm{D}_{2} \mathrm{O}$ ice $\mathrm{I} h$. An ice-free 'structural' dataset with excellent counting statistics was therefore obtained after warming to $280 \mathrm{~K}$. Two $100 \mathrm{~ms}$-wide time-of-flight data-acquisition windows were measured consecutively: $30-130 \mathrm{~ms}$ measured for $2 \mathrm{~h} 25 \mathrm{~m}(104 \mu \mathrm{A} \mathrm{h})$; $100-200 \mathrm{~ms}$ measured for $56 \mathrm{~m}(40 \mu \mathrm{A} \mathrm{h})$. In the instrument's highest resolution backscattering detector banks $(2 \theta=158$ $176^{\circ}$ ) these time windows provide $d$-spacing coverage - after trimming noisier data from the window edges - from 0.65$3.95 \AA$ (Fig. 8). Data were focussed to a common scattering angle $\left(2 \theta=168.3^{\circ}\right)$, normalized to the incident spectrum and corrected for instrument efficiency by reference to a $\mathrm{V}: \mathrm{Nb}$ null-scattering standard using the Mantid suite of powder diffraction algorithms (Mantid, 2013; Arnold et al., 2014).

Since it was clear that the 'heavy' atom structure reported by Meuthen \& von Stackelberg (1960) was not correct, the data were treated $a b$ initio as an unknown. The powder data were indexed using DICVOL06 (Louër \& Boultif, 2007) and examined for systematic absences. This confirmed the crystal system, lattice parameters and space-group assignment of Meuthen \& von Stackelberg (1960), Pbcn, to be correct.

Structure solution was done using the parallel tempering algorithm in FOX, version 1.9.7.1 (Favre-Nicolin \& Černý,
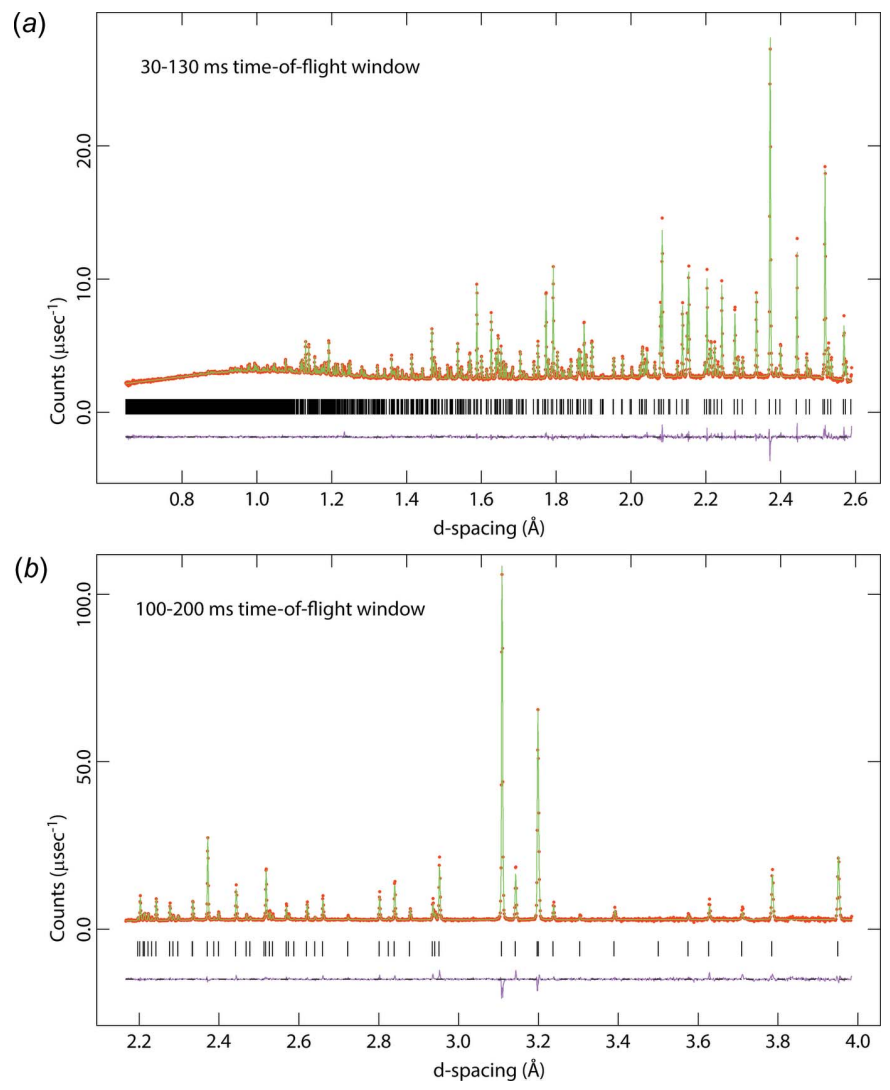

Figure 8

Neutron powder-diffraction data (red circles) measured from phenol hemihydrate at $280 \mathrm{~K}$ using HRPD's 30-130 ms time-of-flight window (a) and the 100-200 ms TOF window $(b)$. The green line indicates the structural model fit and the purple trace underneath is the difference profile. Black tick marks denote the positions of Bragg peaks.
2002, 2004), optimizing the position and orientation of a rigid 'ideal' phenol molecule $(\mathrm{C}-\mathrm{C}=1.390 \AA, \mathrm{C}-\mathrm{O}=1.375 \AA$, $\mathrm{C}-\mathrm{D}=1.085 \AA$, and $\mathrm{O}-\mathrm{D}=0.990 \AA$; all internal angles of the aromatic ring $=120^{\circ}$ and $\mathrm{C}-\mathrm{O}-\mathrm{D}=109^{\circ}$ ) in order to minimize the difference between the observed and calculated diffraction pattern. In twenty runs of $1 / 2$ million trials each, the minimizations consistently produced identical packing arrangements of the phenol molecules, differing from one another only in the position of the origin. Difference-Fourier maps phased on these structures revealed nuclear scattering density around the $4 c$ sites that corresponded to the bridging water molecule. Subsequent addition of a water molecule to this site and relaxation of the phenol molecule's internal degrees of freedom provided a solution with the lowest overall cost function, which formed the basis for further analysis.

The trial structure was refined against the $280 \mathrm{~K}$ neutron powder dataset using GSAS/Expgui (Larsen \& Von Dreele, 2000; Toby, 2001), initially with quite stiff bond-length restraints, and isotropic displacement parameter shifts of similar atoms constrained to be equal. As the refinement progressed, both the bond-length restraints and $U_{\text {iso }}$ constraints were turned off and all atoms were freely refined with anisotropic displacement parameters. The fit to the data collected at $280 \mathrm{~K}$ is shown in Fig. 8.

Additional data were collected in $10 \mathrm{~K}$ increments on cooling to $10 \mathrm{~K}$; each datum involved ramping down the setpoint at $3 \mathrm{~K} \mathrm{~min}^{-1}$, followed by an equilibration dwell time of $10 \mathrm{~m}$ after reaching the set-point, and then a measurement duration of $21 \mathrm{~m}(15 \mu \mathrm{A} \mathrm{h})$. These data reveal not only the presence of Bragg peaks from $\mathrm{D}_{2} \mathrm{O}$ ice $\mathrm{I} h$, but also the substantial broadening of peaks from phenol hemihydrate. The data shown in Fig. 9 were used to obtain the lattice

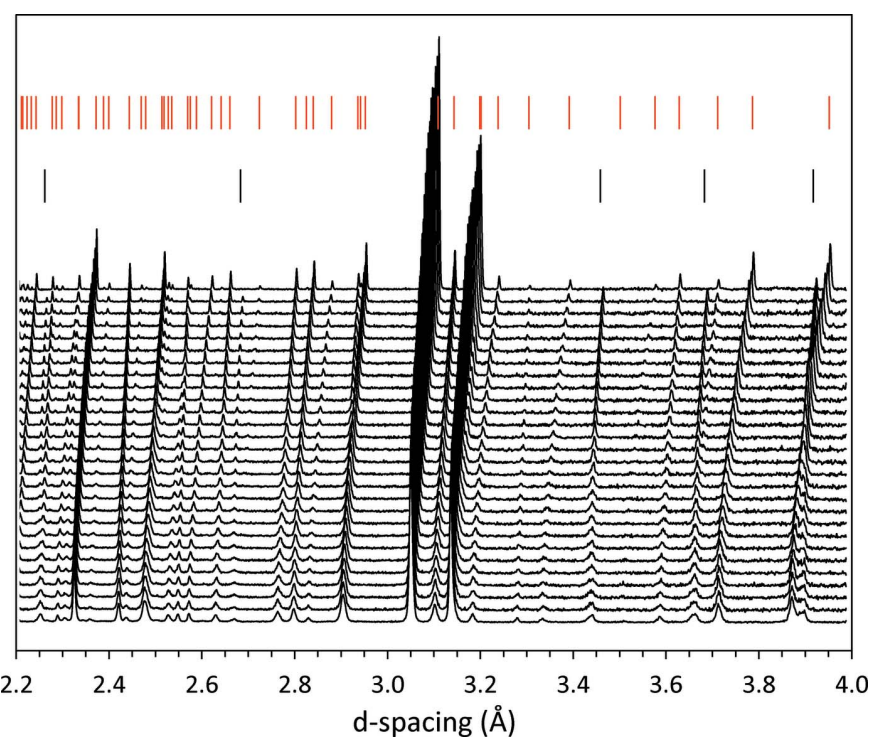

Figure 9

Stack-plot of neutron powder-diffraction data measured on cooling in $10 \mathrm{~K}$ increments from $280 \mathrm{~K}$ (top) to $10 \mathrm{~K}$ (bottom). Red tick marks indicate the Bragg reflections of phenol hemihydrate at $280 \mathrm{~K}$ and the black tick marks show the positions of reflections from ice $\mathrm{I} h$ at $270 \mathrm{~K}$. Note the broadening of all peaks that becomes substantial below $\sim 140 \mathrm{~K}$. 
Table 2

Experimental details.

\begin{tabular}{ll}
\hline Crystal data & \\
Chemical formula & $\mathrm{C}_{6} \mathrm{D}_{6} \mathrm{O} \cdot 0.5 \mathrm{D}_{2} \mathrm{O}$ \\
$M_{\mathrm{r}}$ & 110.16 \\
Crystal system, space group & Orthorhombic, Pbcn \\
Temperature (K) & 280 \\
$a, b, c(\AA)$ & $13.21570(2), 10.89240(2)$, \\
$V\left(\AA^{3}\right)$ & $7.902113(15)$ \\
$Z$ & $1137.52(1)$ \\
Radiation type & 8 \\
Specimen shape, size $(\mathrm{mm})$ & Neutron \\
& Cuboid, $18 \times 23 \times 10$ \\
Data collection & \\
Diffractometer & HRPD, High Resolution Neutron \\
& Powder Diffractometer \\
Specimen mounting & Aluminium-framed slab can with \\
& vanadium windows, shielded \\
& with Gd foil \\
Data collection mode & Reflection \\
Scan method & Time of flight \\
$2 \theta$ values $\left({ }^{\circ}\right)$ & $2 \theta_{\text {fixed }}=168.329$ \\
Distance from source to specimen & 95000 \\
$(\mathrm{~mm})$ & \\
Distance from specimen to & 965 \\
$\quad$ detector (mm) & \\
& \\
Refinement & \\
$R$ factors and goodness of fit & $R_{\mathrm{p}}=0.035, R_{\mathrm{wp}}=0.019$, \\
& $R_{\text {exp }}=0.045, R\left(F^{2}\right)=0.46850$, \\
No. of parameters & 153 \\
\hline
\end{tabular}

Computer programs: HRPD DAE3, IBEX (Akeroyd et al., 2018), Mantid (Arnold et al., 2014: Mantid, 2013), FOX (Favre-Nicolin \& Černý, 2002, 2004), GSAS (Larsen \& Von Dreele, 2000), DIAMOND (Putz \& Brandenburg, 2006), VESTA (Momma \& Izumi, 2011) and publCIF (Westrip, 2010).

parameters given in supplementary Table S1 and analysed in section 5 above.

\section{Synthesis and crystallization}

Crystalline phenol- $d_{6}$ (Sigma Aldrich 176060, 99 atom \% D) was mixed with liquid $\mathrm{D}_{2} \mathrm{O}$ (Aldrich $151882,99.9$ atom \% D) to form an aqueous solution with a composition equivalent to the stoichiometry of phenol- $d_{6}$ hemideuterate (90.910 wt.\% phenol- $d_{6}$ ). This liquid was decanted dropwise with a glass pipette directly into liquid nitrogen, forming pinkish-white solid spherules 3-5 $\mathrm{mm}$ in diameter. These were transferred into a glass vial and stored in a freezer at $\sim 255 \mathrm{~K}$ for several days. Directly prior to the start of the measurements, the contents of the vial were ground to a fine pale-pink powder under liquid nitrogen and transferred into a nitrogen-chilled sample holder. The sample container consisted of a solid aluminium alloy frame with a cuboid central cavity $18 \mathrm{~mm} \mathrm{x}$ $23 \mathrm{~mm}(w \times h$ perpendicular to the incident neutron beam $) \times$ $10 \mathrm{~mm}$ (depth parallel to the incident beam). The open front and back sides of the sample were covered with vanadium foil windows $(125 \mu \mathrm{m}$ thick $)$, held in place with stainless steel frames and sealed with indium wire. Exposed $\mathrm{Al}$ and steel around the 'front' vanadium window were masked with Gd and $\mathrm{Cd}$ foils. Sample temperatures were monitored with a $\mathrm{RhFe}$ thermocouple embedded in the $\mathrm{Al}$ frame; active heating was generated by a Watlow Firerod cartridge heater embedded in the opposite side of the sample holder. The whole assembly was mounted on a centre stick and inserted in a closed-cycle refrigerator (CCR) in order to carry out variable-temperature measurements.

\section{Acknowledgements}

The author thanks the STFC ISIS neutron and muon spallation source for beam-time access, RB1920009 (doi:10.5286/ ISIS.E.RB1920009) and STFC's SCARF high-performance computing cluster for the resources needed to carry out the DFT calculations.

\section{References}

Akeroyd, F. A., Baker, K. V. L., Clarke, M. J., Holt, J. R., Howells, G. D., Keymer, D. P., Löhnert, T., Moreton-Smith, C. M., Oram, D. E., Potter, A., Rey, I. H., Willemson, T. A. \& Wood, K. (2018). J. Phys. Conf. Ser. 1021, 012019.

Alexeev, V. Th. (1883). Zh. Russ. Fiz. Khim. Obshch, 15, 412-413.

Allan, D. R., Clark, S. J., Dawson, A., McGregor, P. A. \& Parsons, S. (2002). Acta Cryst. B58, 1018-1024.

André, D., Fourme, R. \& Renaud, M. (1971). Acta Cryst. B27, 23712380.

Arnold, O., Bilheux, J. C., Borreguero, J. M., Buts, A., Campbell, S. I., Chapon, L., Doucet, M., Draper, N., Ferraz Leal, R., Gigg, M. A., Lynch, V. E., Markvardsen, A., Mikkelson, D. J., Mikkelson, R. L., Miller, R., Palmen, K., Parker, P., Passos, G., Perring, T. G., Peterson, P. F., Ren, S., Reuter, M. A., Savici, A. T., Taylor, J. W., Taylor, R. J., Tolchenov, R., Zhou, W. \& Zikovsky, J. (2014). Nucl. Instrum. Methods Phys. Res. A, 764, 156-166.

Binns, J., Healy, M. R., Parsons, S. \& Morrison, C. A. (2014). Acta Cryst. B70, 259-267.

Biswas, S. G. (1958). Acta Cryst. 11, 882-884.

Calvert, F. C. (1865). J. Chem. Soc. 18, 66-70.

Chantrapromma, S., Jansrisewangwong, P., Chanawanno, K. \& Fun, H.-K. (2011). Acta Cryst. E67, o2221-o2222.

Clark, S. J., Segall, M. D., Pickard, C. J., Hasnip, P. J., Probert, M. I. J., Refson, K. \& Payne, M. C. (2005). Z. Kristallogr. 220, 567-570.

Demirtaş, G., Dege, N. \& Büyükgüngör, O. (2011). Acta Cryst. E67, o1509-o1510.

Favre-Nicolin, V. \& Černý, R. (2002). J. Appl. Cryst. 35, 734-743.

Favre-Nicolin, V. \& Černý, R. (2004). Z. Kristallogr. 219, 847-856.

Fortes, A. D. (2019). Structures of phenol-ammonia and phenol-water compounds. STFC ISIS Neutron and Muon Source, RB1920009. https://doi.org/10.5286/ISIS.E.RB1920009.

Grimme, S. (2006). J. Comput. Chem. 27, 1787-1799.

Groom, C. R., Bruno, I. J., Lightfoot, M. P. \& Ward, S. C. (2016). Acta Cryst. B72, 171-179.

Hohenberg, P. \& Kohn, W. (1964). Phys. Rev. 136, B864-B871.

Ibberson, R. M. (2009). Nucl. Instrum. Methods Phys. Res. A, 600, 4749.

Kohn, W. \& Sham, L. J. (1965). Phys. Rev. 140, A1133-A1138.

Larsen, A. C. \& Von Dreele, R. B. (2000). General Structure Analysis System (GSAS). Los Alamos National Laboratory Report LAUR 86-748, Los Alamos, New Mexico, USA.

Li, H.-P., Yang, Y.-X. \& Ng, S. W. (2010). Acta Cryst. E66, o2867.

Louër, D. \& Boultif, A. (2007). Z. Kristallogr. Suppl. pp. 191-196.

Mantid (2013). Manipulation and Analysis Toolkit for Instrument Data; Mantid Project. http://dx.doi.org/10.5286/SOFTWARE/ MANTID.

McKinnon, J. J., Jayatilaka, D. \& Spackman, M. A. (2007). Chem. Commun. pp. 3814-3816.

Merz, K. (2006). Cryst. Growth Des. 6, 1615-1619. 
Meuthen, B. \& von Stackelberg, M. (1960). Z. Elektrochem, 64, 387390.

Momma, K. \& Izumi, F. (2011). J. Appl. Cryst. 44, 1272-1276.

Nath, N. K. \& Naumov, P. (2015). Maced. J. Chem. Chem. Eng. 34, 6366.

Pachler, K. \& Von Stackelberg, M. (1963). Z. Kristallogr. 119, 15-29.

Paternò, E. \& Ampola, G. (1897). Gazz. Chim. Ital. 27, 481-536.

Payne, M. C., Teter, M. P., Allan, D. C., Arias, T. A. \& Joannopoulos, J. D. (1992). Rev. Mod. Phys. 64, 1045-1097.

Perdew, J. P., Burke, K. \& Ernzerhof, M. (1996). Phys. Rev. Lett. 77, 3865-3868.

Perdew, J. P., Burke, K. \& Ernzerhof, M. (1997). Phys. Rev. Lett. 78, 1396.

Pfrommer, B. G., Côté, M., Louie, S. G. \& Cohen, M. L. (1997). J. Comput. Phys. 131, 233-240.

Putz, H. \& Brandenburg, K. (2006). Diamond - Crystal and Molecular Structure Visualization. Crystal Impact - GbR, Kreuzherrenstr. 102, 53227 Bonn, Germany. (http://www.crystalimpact.com/diamond)

Rhodes, F. H. \& Markley, A. L. (1921). J. Phys. Chem. 25, 527-534.

Segall, M. D., Lindan, P. J. D., Probert, M. J., Pickard, C. J., Hasnip, P. J., Clark, S. J. \& Payne, M. C. (2002). J. Phys. Condens. Matter, 14, 2717-2744.
Smith, R. A. (1932). Mikrochemie, 11, 227-236.

Smits, A. \& Maarse, J. (1911). Kong. Nederl. Akad. Wetensch, 14, 192195.

Spackman, M. A. \& Jayatilaka, D. (2009). CrystEngComm, 11, 1932.

Thomas, S. P., Sathishkumar, R. \& Guru Row, T. N. (2015). Chem. Commun. 51, 14255-14258.

Tkatchenko, A., DiStasio, R. A., Car, R. \& Scheffler, M. (2012). Phys. Rev. Lett. 108, 236402.

Tkatchenko, A. \& Scheffler, M. (2009). Phys. Rev. Lett. 102, 073005073008.

Toby, B. H. (2001). J. Appl. Cryst. 34, 210-213.

Turner, M. J., McKinnon, J. J., Wolff, S. K., Grimwood, D. J., Spackman, P. R., Jayatilaka, D. \& Spackman, M. A. (2017). CrystalExplorer17. University of Western Australia. (http://hirshfeldsurface. net)

Westrip, S. P. (2010). J. Appl. Cryst. 43, 920-925.

Zavodnik, V. E., Bel'skii, V. K. \& Zorkii, P. M. (1988). J. Struct. Chem. 28, 793-795.

Zhang, X. Q., Mou, X. F., Mao, N., Hao, J. J., Liu, M., Zheng, J. Y., Wang, C. Y., Gu, Y. C. \& Shao, C. L. (2018). Eur. J. Med. Chem. 146, 232-244. 


\section{supporting information}

Acta Cryst. (2020). E76, 1062-1069 [https://doi.org/10.1107/S2056989020007719]

Phenol hemihydrate: redetermination of the crystal structure by neutron powder diffraction, Hirshfeld surface analysis and characterization of the thermal expansion

\section{A. Dominic Fortes}

Computing details

Program(s) used to solve structure: FOX (Favre-Nicolin \& Černý, 2002, 2004).

Phenol- $d_{6}$ hemideuterate

Crystal data

$\mathrm{C}_{6} \mathrm{D}_{6} \mathrm{O} \cdot 0.5 \mathrm{D}_{2} \mathrm{O}$

$M_{r}=110.16$

Orthorhombic, $\mathrm{Pbcn}$

Hall symbol: -P 2n 2ab

$a=13.21570(2) \AA$

$b=10.89240(2) \AA$

$c=7.902113(15) \AA$

$$
\begin{aligned}
& V=1137.52(1) \AA^{3} \\
& Z=8 \\
& F(000)=440.0 \\
& D_{\mathrm{x}}=1.286 \mathrm{Mg} \mathrm{m}^{-3} \\
& \text { Melting point: } 289 \mathrm{~K} \\
& T=280 \mathrm{~K} \\
& \text { Particle morphology: fine powder }
\end{aligned}
$$

Fractional atomic coordinates and isotropic or equivalent isotropic displacement parameters $\left(\AA^{2}\right)$

\begin{tabular}{lllll}
\hline & $x$ & $y$ & $z$ & $U_{\text {iso }} / U_{\text {eq }}$ \\
\hline C1 & $0.2611(2)$ & $0.5384(3)$ & $0.3681(4)$ & 0.06249 \\
D1 & $0.2246(2)$ & $0.4652(4)$ & $0.2981(5)$ & 0.09617 \\
C2 & $0.3664(2)$ & $0.5482(4)$ & $0.3650(5)$ & 0.07906 \\
D2 & $0.4099(3)$ & $0.4827(5)$ & $0.2967(6)$ & 0.12157 \\
C3 & $0.4154(3)$ & $0.6429(3)$ & $0.4484(5)$ & 0.07808 \\
D3 & $0.4947(3)$ & $0.6522(4)$ & $0.4493(6)$ & 0.11566 \\
C4 & $0.3580(3)$ & $0.7277(4)$ & $0.5342(5)$ & 0.07491 \\
D4 & $0.3925(3)$ & $0.8022(5)$ & $0.6060(7)$ & 0.13499 \\
C5 & $0.2519(3)$ & $0.7205(4)$ & $0.5382(4)$ & 0.06855 \\
D5 & $0.2059(3)$ & $0.7837(4)$ & $0.6052(5)$ & 0.10795 \\
C6 & $0.2043(2)$ & $0.6255(3)$ & $0.4538(4)$ & 0.06001 \\
O1 & $0.1012(3)$ & $0.6199(4)$ & $0.4663(6)$ & 0.07452 \\
D7 & $0.0767(3)$ & $0.5638(5)$ & $0.3808(6)$ & 0.08765 \\
Ow1 & 0.0 & $0.4584(7)$ & 0.25 & 0.06156 \\
Dw1 & $0.0326(3)$ & $0.4065(4)$ & $0.1647(6)$ & 0.09388 \\
\hline
\end{tabular}


Atomic displacement parameters $\left(\AA^{2}\right)$

\begin{tabular}{lllllll}
\hline & $U^{11}$ & $U^{22}$ & $U^{33}$ & $U^{12}$ & $U^{13}$ & $U^{23}$ \\
\hline C1 & $0.075(3)$ & $0.047(2)$ & $0.066(3)$ & $-0.0009(17)$ & $-0.0008(18)$ & $-0.0091(17)$ \\
D1 & $0.075(3)$ & $0.092(3)$ & $0.121(4)$ & $-0.004(2)$ & $0.0041(18)$ & $-0.049(3)$ \\
C2 & $0.051(3)$ & $0.081(3)$ & $0.105(4)$ & $0.013(2)$ & $0.013(2)$ & $0.001(2)$ \\
D2 & $0.079(3)$ & $0.140(5)$ & $0.145(4)$ & $0.036(3)$ & $0.008(2)$ & $-0.046(3)$ \\
C3 & $0.052(2)$ & $0.088(3)$ & $0.094(3)$ & $-0.010(2)$ & $-0.011(2)$ & $0.001(2)$ \\
D3 & $0.051(2)$ & $0.135(4)$ & $0.161(4)$ & $-0.003(2)$ & $-0.005(3)$ & $-0.005(3)$ \\
C4 & $0.074(3)$ & $0.065(3)$ & $0.086(3)$ & $-0.012(2)$ & $0.008(2)$ & $-0.023(2)$ \\
D4 & $0.111(3)$ & $0.096(4)$ & $0.198(6)$ & $-0.025(3)$ & $0.003(3)$ & $-0.041(3)$ \\
C5 & $0.063(3)$ & $0.077(3)$ & $0.066(3)$ & $0.0008(18)$ & $0.0201(18)$ & $-0.009(2)$ \\
D5 & $0.076(2)$ & $0.099(3)$ & $0.149(4)$ & $-0.005(2)$ & $0.021(3)$ & $-0.059(2)$ \\
C6 & $0.059(2)$ & $0.059(2)$ & $0.0615(19)$ & $-0.0039(18)$ & $0.0016(18)$ & $0.0018(15)$ \\
O1 & $0.055(3)$ & $0.085(4)$ & $0.083(4)$ & $0.002(2)$ & $0.009(2)$ & $-0.024(3)$ \\
D7 & $0.071(3)$ & $0.102(4)$ & $0.090(3)$ & $0.011(2)$ & $-0.001(2)$ & $-0.012(2)$ \\
Ow1 & $0.067(4)$ & $0.076(5)$ & $0.042(4)$ & 0.0 & $0.005(3)$ & 0.0 \\
Dw1 & $0.078(2)$ & $0.086(4)$ & $0.118(4)$ & $0.009(2)$ & $0.004(2)$ & $-0.019(2)$ \\
& & & & & & \\
\hline
\end{tabular}

Geometric parameters $\left(\AA,{ }^{\circ}\right)$

\begin{tabular}{|c|c|c|c|}
\hline $\mathrm{C} 1-\mathrm{D} 1$ & $1.084(4)$ & $\mathrm{D} 5-\mathrm{C} 5$ & $1.060(4)$ \\
\hline $\mathrm{C} 1-\mathrm{C} 2$ & $1.396(4)$ & D5-C6 & $2.098(5)$ \\
\hline $\mathrm{C} 1-\mathrm{C} 6$ & $1.387(4)$ & $\mathrm{C} 6-\mathrm{C} 1$ & $1.387(4)$ \\
\hline $\mathrm{D} 1-\mathrm{C} 1$ & $1.084(4)$ & $\mathrm{C} 6-\mathrm{C} 5$ & $1.382(4)$ \\
\hline $\mathrm{C} 2-\mathrm{C} 1$ & $1.396(4)$ & $\mathrm{C} 6-\mathrm{D} 5$ & $2.098(5)$ \\
\hline $\mathrm{C} 2-\mathrm{D} 2$ & $1.063(4)$ & $\mathrm{C} 6-\mathrm{O} 1$ & $1.368(5)$ \\
\hline $\mathrm{C} 2-\mathrm{C} 3$ & $1.385(5)$ & C6-D7 & $1.905(5)$ \\
\hline $\mathrm{D} 2-\mathrm{C} 2$ & $1.063(4)$ & $\mathrm{O} 1-\mathrm{C} 6$ & $1.368(5)$ \\
\hline $\mathrm{C} 3-\mathrm{C} 2$ & $1.385(5)$ & $\mathrm{O} 1-\mathrm{D} 7$ & $0.967(4)$ \\
\hline $\mathrm{C} 3-\mathrm{D} 3$ & $1.053(3)$ & $\mathrm{O} 1-\mathrm{Dw} 1^{\mathrm{i}}$ & $1.833(6)$ \\
\hline $\mathrm{C} 3-\mathrm{C} 4$ & $1.374(4)$ & D7-C6 & $1.905(5)$ \\
\hline D3-C3 & $1.053(3)$ & $\mathrm{D} 7-\mathrm{O} 1$ & $0.967(4)$ \\
\hline D3-C4 & $2.096(5)$ & D7-Ow1 & $1.847(7)$ \\
\hline $\mathrm{C} 4-\mathrm{C} 3$ & $1.374(4)$ & Ow1-D7 & $1.847(7)$ \\
\hline C4-D3 & $2.096(5)$ & Ow1-D7 $7^{\mathrm{ii}}$ & $1.847(7)$ \\
\hline $\mathrm{C} 4-\mathrm{D} 4$ & $1.091(4)$ & Ow1-Dw1 & $0.980(4)$ \\
\hline $\mathrm{C} 4-\mathrm{C} 5$ & $1.405(4)$ & Ow1-Dw $1^{\text {ii }}$ & $0.980(4)$ \\
\hline D4-C4 & $1.091(4)$ & Dw1-O $1^{\mathrm{iii}}$ & $1.833(6)$ \\
\hline $\mathrm{C} 5-\mathrm{C} 4$ & $1.405(4)$ & Dw1-Ow1 & $0.980(4)$ \\
\hline $\mathrm{C} 5-\mathrm{D} 5$ & $1.060(4)$ & Dw1-Dw $1^{\text {ii }}$ & $1.600(8)$ \\
\hline $\mathrm{C} 5-\mathrm{C} 6$ & $1.382(4)$ & & \\
\hline $\mathrm{D} 1-\mathrm{C} 1-\mathrm{C} 2$ & $119.4(4)$ & $\mathrm{C} 3-\mathrm{C} 4-\mathrm{C} 5$ & $121.7(4)$ \\
\hline $\mathrm{D} 1-\mathrm{C} 1-\mathrm{C} 6$ & $120.8(4)$ & $\mathrm{D} 4-\mathrm{C} 4-\mathrm{C} 5$ & $116.6(4)$ \\
\hline $\mathrm{C} 2-\mathrm{C} 1-\mathrm{C} 6$ & $119.7(4)$ & $\mathrm{C} 4-\mathrm{C} 5-\mathrm{D} 5$ & $123.2(4)$ \\
\hline $\mathrm{C} 1-\mathrm{C} 2-\mathrm{D} 2$ & $119.7(5)$ & $\mathrm{C} 4-\mathrm{C} 5-\mathrm{C} 6$ & $119.0(4)$ \\
\hline $\mathrm{C} 1-\mathrm{C} 2-\mathrm{C} 3$ & $121.0(4)$ & $\mathrm{D} 5-\mathrm{C} 5-\mathrm{C} 6$ & $117.8(4)$ \\
\hline
\end{tabular}




$\begin{array}{llll}\mathrm{D} 2-\mathrm{C} 2-\mathrm{C} 3 & 119.3(4) & \mathrm{C} 1-\mathrm{C} 6-\mathrm{C} 5 & 120.1(3) \\ \mathrm{C} 2-\mathrm{C} 3-\mathrm{D} 3 & 122.7(5) & \mathrm{C} 1-\mathrm{C} 6-\mathrm{O} 1 & 123.0(4) \\ \mathrm{C} 2-\mathrm{C} 3-\mathrm{C} 4 & 118.5(4) & \mathrm{C} 5-\mathrm{C} 6-\mathrm{O} 1 & 116.9(4) \\ \mathrm{D} 3-\mathrm{C} 3-\mathrm{C} 4 & 118.8(5) & \mathrm{C} 6-\mathrm{O} 1-\mathrm{D} 7 & 108.2(5) \\ \mathrm{C} 3-\mathrm{C} 4-\mathrm{D} 4 & 121.7(5) & \mathrm{Dw} 1-\mathrm{Ow} 1-\mathrm{Dw} 1^{\mathrm{ii}} & 109.4(10)\end{array}$

Symmetry codes: (i) $x,-y+1, z+1 / 2$; (ii) $-x, y,-z+1 / 2$; (iii) $x,-y+1, z-1 / 2$.

Hydrogen-bond geometry $\left(A,{ }^{\circ}\right)$

$\mathrm{Cg}$ is the centroid of the $\mathrm{C} 1-\mathrm{C} 6$ ring located at $(0.310,0.634,0.451)$.

\begin{tabular}{lllll}
\hline$D-\mathrm{H} \cdots A$ & $D-\mathrm{H}$ & $\mathrm{H} \cdots A$ & $D \cdots A$ & $D-\mathrm{H} \cdots A$ \\
\hline $\mathrm{O} 1-\mathrm{D} 7 \cdots \mathrm{Ow}$ & $0.97(1)$ & $1.85(1)$ & $2.793(7)$ & $165(1)$ \\
Ow-Dwii $\cdots 1^{\mathrm{iv}}$ & $0.98(1)$ & $1.83(1)$ & $2.746(5)$ & $154(1)$ \\
$\mathrm{C} 1-\mathrm{D} 1 \cdots C g^{\mathrm{iii}}$ & $1.08(1)$ & 3.15 & 3.844 & 123 \\
$\mathrm{C} 2-\mathrm{D} 2 \cdots C g^{\mathrm{iii}}$ & $1.06(1)$ & 3.29 & 3.897 & 118 \\
$\mathrm{C} 5-\mathrm{D} 5 \cdots \mathrm{Cg}^{\mathrm{v}}$ & $1.06(1)$ & 2.89 & 3.719 & 136
\end{tabular}

Symmetry codes: (ii) $-x, y,-z+1 / 2$; (iii) $x,-y+1, z-1 / 2$; (iv) $-x,-y+1,-z+1$; (v) $-x+1 / 2,-y+3 / 2, z+1 / 2$. 